\title{
Resección laparoscópica de metástasis intestinales de melanoma maligno
}

\author{
Pablo Marcos S. ${ }^{1}$, Martín Bailón C. ${ }^{1}$, Miguel Toledano T. ${ }^{1}$ y David Pacheco S. ${ }^{1}$
}

\section{'Servicio de Cirugía General y Digestiva, Hospital Universitario Río Hortega. Valladolid, España. \\ Recibido el 1 de diciembre de 2019 y aceptado para publicación el 6 de enero de

Correspondencia a: Dr. Pablo Marcos S. pmarcosan@gmail.com

\section{Laparoscopic resection of malignant melanoma intestinal metastasis}

\begin{abstract}
Aim: Cutaneous melanoma has a high metastatic potential, being the most frequent extra-abdominal source of small bowel metastasis. The diagnosis of gastrointestinal metastases is often an expression of advanced disease, with an average survival of 6-9 months. Materials and Method: We herein present the case of a 63-year-old male patient diagnosed with cutaneous melanoma who arrived to the emergency department complaining of abdominal pain and constipation. An abdominal CT scan was performed, it revealed two metastatic lesions in the small bowel which marked the mechanical obstruction. Results: Patient underwent a laparoscopy and both involved segments were removed. Pathology exam confirmed the diagnosis of melanoma metastases. Discussion: Surgery excision is the treatment of choice in patients with small bowel metastases from melanoma. Surgical management can improve the prognosis and it would be indicated in cases of single metastases or symptomatic patients with a palliative intention. Minimally invasive approach provides similar oncological results as conventional laparotomy. Small bowel obstruction due to metastases of malignant melanoma is extremely rare.
\end{abstract}

Key words: melanoma; intestinal obstruction; metastasectomy.

\section{Resumen}

Objetivos: El melanoma cutáneo presenta un alto potencial metastásico y constituye la fuente extraabdominal más frecuente de lesión del intestino delgado. El diagnóstico de metástasis gastrointestinales es a menudo una expresión de enfermedad avanzada, con una supervivencia media de entre 6 y 9 meses. Materiales y Método: Presentamos el caso de un paciente varón de 63 años diagnosticado de melanoma cutáneo que acudió a urgencias por dolor abdominal y estreñimiento. Se realizó una TC abdominal donde se informó de la existencia de 2 lesiones metastásicas a nivel de intestino delgado que condicionaban oclusión intestinal. Resultados: El paciente fue intervenido quirúrgicamente bajo abordaje laparoscópico con resección de los dos segmentos intestinales afectos y anastomosis intracorpórea. El informe histopatológico confirmó que se trataban de metástasis de melanoma. Discusión: La oclusión intestinal por metástasis de melanoma maligno es muy infrecuente. La cirugía es el tratamiento de elección en pacientes con metástasis intestinales de melanoma. El tratamiento quirúrgico puede mejorar el pronóstico y estaría indicado casos de metástasis únicas o pacientes sintomáticos con intención paliativa. El abordaje mínimamente invasivo ofrece resultados oncológicos similares a la laparotomía.

Palabras clave: melanoma; obstrucción intestinal; metastasectomía.

\section{Introducción}

El melanoma cutáneo presenta un alto potencial metastásico y se trata de la fuente extraabdominal más frecuente de lesiones en el intestino delgado. Se encuentran metástasis en el tracto gastrointestinal en el $60 \%$ de los pacientes que mueren a causa de esta enfermedad, y en el 50\%-71\% afectan a intestino delgado ${ }^{1}$. El diagnóstico de metástasis gas- trointestinales de melanoma maligno es a menudo una expresión de enfermedad avanzada y presenta un mal pronóstico, con una supervivencia media de entre 6 y 9 meses y una tasa de supervivencia a 5 años menor del $10 \%{ }^{2}$. Presentamos el caso de un paciente diagnosticado de melanoma cutáneo estadio IV con metástasis intestinales que precisó intervención quirúrgica urgente por obstrucción intestinal. 


\section{Materiales y Método}

Paciente varón de 63 años diagnosticado de melanoma cutáneo sin enfermedad a distancia en el seguimiento oncológico. Como antecedentes personales presentaba hipertensión arterial e infarto de miocardio, sin intervenciones quirúrgicas previas. El paciente debutó hace un año con un melanoma cutáneo en el cuarto dedo del pie izquierdo. La biopsia reveló que se trataba de un melanoma nodular polipoide en fase de crecimiento vertical con un nivel $\mathrm{V}$ de Clark, un índice de Breslow de $8 \mathrm{~mm}$ y con gen BRAF nativo. En el estudio de estadificación se detectaron nódulos bilaterales pulmonares compatibles con metástasis, por lo que se clasificó como un pT4b pN1a M1x. Tras la biopsia y estadificación del melanoma se inició tratamiento adyuvante con 16 ciclos de Nivolumab durante 10 meses. Durante este periodo el paciente presentó respuesta completa con desaparición de las metástasis pulmonares, sin detectarse nuevas lesiones a distancia.

El paciente acudió a urgencias por un dolor abdominal difuso y estreñimiento de 5 días de evolución, acompañados de distensión abdominal, náuseas y vómitos, sin fiebre. El último ciclo de terapia adyuvante con Nivolumab se había administrado hacía 6 meses. A la exploración abdominal presentaba distensión y timpanismo, con dolor difuso a la palpación, sin signos de irritación peritoneal. No se palpaban hernias ni otras masas. La analítica sanguínea no reveló alteraciones significativas. En la radiografía de abdomen se visualizó una dilatación de intestino delgado sin gas en la ampolla rectal. Se realizó una TC abdominal en que se describían dos lesiones sólidas en íleon proximal, sugerentes de lesiones metastásicas, que condicionaban una repercusión obstructiva con dilatación de las asas yeyunales. Una masa polipoidea de $5 \times 3,5 \times 3 \mathrm{~cm}$, hipercaptante, de crecimiento intraluminal (Figura 1A) $\mathrm{y}$, proximalmente a unos $4 \mathrm{~cm}$, un engrosamiento hipercaptante mural asimétrico de unos 2 $\mathrm{cm}$ (Figura 1B).

Con el diagnóstico de obstrucción intestinal se decidió intervenir al paciente de forma urgente. La intervención fue realizada mediante abordaje laparoscópico y se identificaron 2 zonas estenóticas retráctiles separadas entre sí unos $70 \mathrm{~cm}$ (Figura 2A). El último control con imagen se correspondía con una tomografía por emisión de positrones de cuerpo entero, realizada 6 meses antes del episodio de obstrucción intestinal, en el que se apreció una respuesta de las metástasis a nivel pulmonar y no se detectaron focos hipermetabólicos significativos a nivel abdominal. Se procedió a realizar una doble resección en cuña de ambas lesiones (Figura 2B) con posterior anastomosis latero-lateral mecánica intracorpórea y cierre de enterostomía con sutura manual. El paciente evolucionó favorablemente

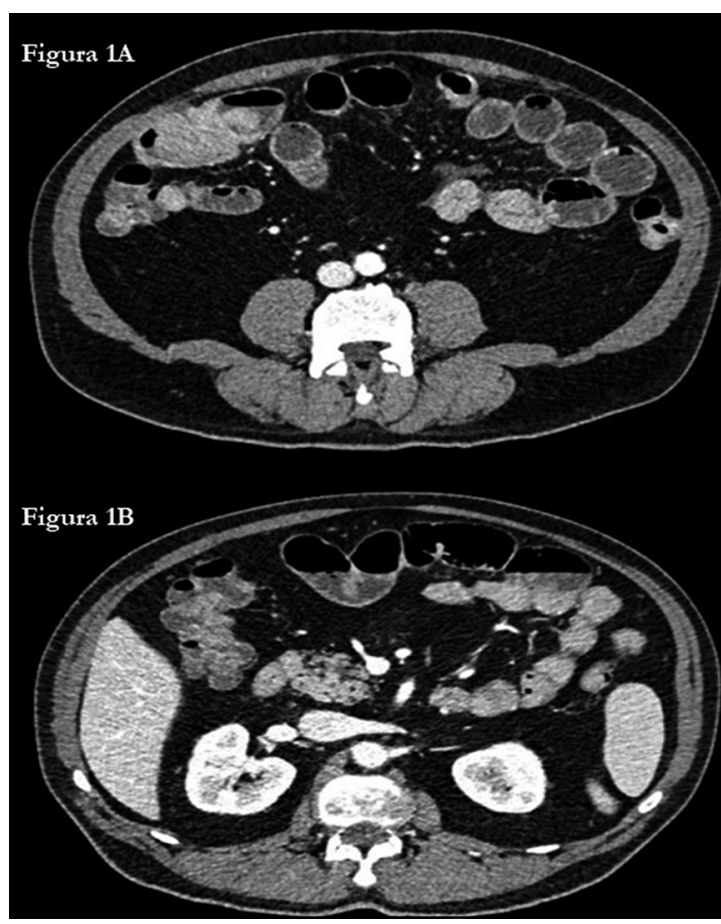

Figura 1. A: Engrosamiento hipercaptante mural asimétrico del asa intestinal, de unos dos centímetros de extensión. B: Masa polipoide, hipercaptante, de $5 \times 3,5 \times 3 \mathrm{~cm}$ de diámetros transverso, anteroposterior y craneocaudal, de crecimiento fundamentalmente intraluminal.

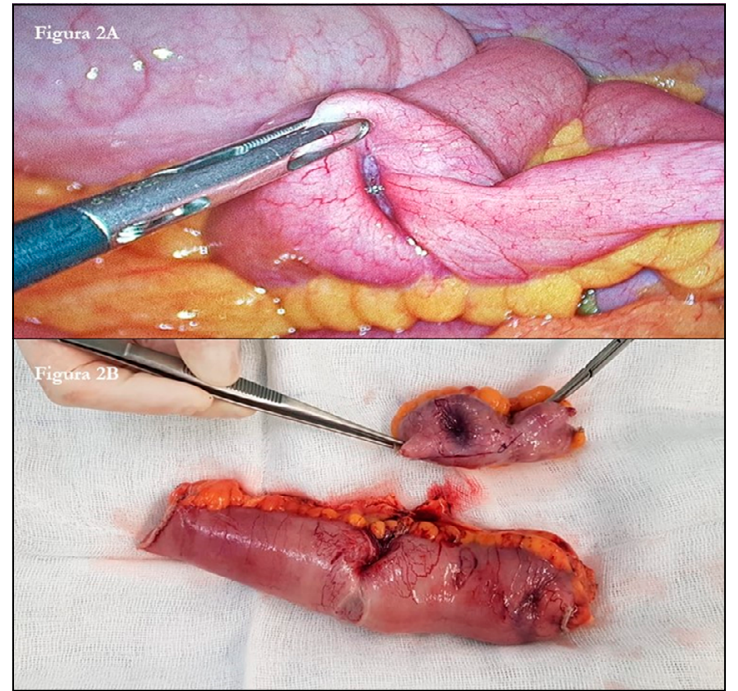

Figura 2. A: Se aprecia una zona estenótica y retráctil condicionando obstrucción a nivel del segmento intestinal afecto. B: Piezas quirúrgicas resecadas en cuña afectadas con lesiones endoluminales que, posteriormente, se confirmaron como metástasis de melanoma maligno. 
con buena tolerancia oral y restablecimiento del tránsito intestinal, siendo dado de alta a la semana de la intervención. El estudio histopatológico reveló metástasis polipoides de melanoma maligno, con bordes de resección libre. Tras la intervención quirúrgica se planteó iniciar un tratamiento adyuvante con Ipilumumab. A los 3 meses de la intervención quirúrgica urgente, por el episodio de obstrucción intestinal, el paciente presentó metástasis cerebrales que condujeron al fallecimiento del paciente.

\section{Discusión}

La primera causa de obstrucción de intestino delgado son las adherencias secundarias a cirugías previas, seguidas de los tumores malignos y las hernias. Los tumores malignos suponen el $20 \%$ de las obstrucciones de intestino delgado, siendo lo más frecuente las lesiones metastásicas en el intestino delgado. El melanoma cutáneo tiene un alto potencial metastásico y es la fuente extraabdominal más frecuente de lesiones en el intestino delgado. El tracto gastrointestinal ocupa el tercer lugar en frecuencia de metástasis a distancia, siendo el intestino delgado el más comúnmente afectado ${ }^{3}$. Sin embargo, únicamente en un 5-6\% de pacientes con melanoma maligno se llega al diagnóstico de metástasis gastrointestinales ${ }^{4}$. El diagnóstico de metástasis gastrointestinales de melanoma maligno es, a menudo, una expresión de enfermedad avanzada y presenta un mal pronóstico. Se debe sospechar la existencia de metástasis gastrointestinales en estos pacientes cuando presenten dolor abdominal, obstrucción intestinal o hemorragia digestiva, habitualmente acompañados de anorexia, adelgazamiento y anemia ${ }^{5}$. Pueden dar lugar a cuadros de oclusión, invaginación o hemorragia digestiva. Pese a todos estos datos, la obstrucción intestinal por metástasis de melanoma es muy infrecuente. El principal factor pronóstico en pacientes con melanoma maligno en estadio IV es la localización de las metástasis a distancia. Aquellas localizadas en pulmón, hígado o tracto gastrointestinal conllevan un pronóstico peor ${ }^{6}$.

Clásicamente, los pacientes con melanoma maligno estadio IV presentaban un pronóstico infausto a corto plazo, con una supervivencia media inferior a 6 meses. Sin embargo, gracias a los avances en las nuevas dianas terapéuticas, se han desarrollado fármacos que permiten aumentar la supervivencia libre de progresión, alcanzándose supervivencias globales superiores a los 2-3 años. Este notable incremento de la supervivencia en pacientes con enfermedad avanzada redefine la importancia del tratamiento quirúrgico de las complicaciones derivadas de la progresión de la enfermedad ${ }^{7}$. El tratamiento quirúrgico de estas lesiones podría estar indicado en pacientes sintomáticos o en pacientes con metástasis únicas con intención curativa. Algunas publicaciones describen que la cirugía puede mejorar el pronóstico en casos de pacientes con metástasis aisladas $^{8}$. El tratamiento quirúrgico se basa en la resección paliativa, para aliviar los síntomas, o en una derivación intestinal en casos de lesiones tan extensas que impidan la resección ${ }^{9}$. Dada la infrecuencia de esta patología no existe evidencia suficiente para recomendar el abordaje mínimamente invasivo, pero se trata de un abordaje seguro, menos agresivo y con resultados oncológicos similares a la laparotomía en estudios existentes en la literatura ${ }^{10}$.

\section{Responsabilidades éticas}

Protección de personas y animales. Los autores declaran que para esta investigación no se han realizado experimentos en seres humanos ni en animales.

Confidencialidad de los datos. Los autores declaran que en este artículo no aparecen datos de pacientes.

Conflictos de interés: no hay.

\section{Bibliografía}

1. Carvajal López F, Muñoz Muñoz E, Rodríguez Alsina X, Salas Caudevilla A, Marco Molina C. Metástasis intestinal de melanoma maligno cutáneo como causa de intususcepción yeyunal recurrente. Cir Esp. 2012;90:473-6.

2. Lens M, Bataille V, Krivokapic Z. Melanoma of the small intestine. Lancet Oncol. 2009;10:516-21.
3. Blecker D, Abraham S, Furth EE, Kochman ML. Melanoma in the gastrointestinal tract. Am J Gastroenterol. 1999;94:3427-33.

4. Dokic M, Badovinac D, Petric M, Trotovsek B. An unusual presentation of metastatic malignant melanoma causing jejuno-jenual intussuception: a case report. J Med Case Rep. 2018;12:337. Published online 2018 Nov 13. doi: 10.1186/s13256018-1887-5.
5. Mantas D, Damaskos C, Garmpis N, Dimitroulis D, Garmpi A, Gogas H. Abdominal emergencies in patients with stage IV melanoma: The role of surgery: A single-centre experience. Antincancer Res. 2018;38:3713-8.

6. Soares F, Brandao P, Inez-Correoia R, Valente V. Small bowel obstruction due to intraluminal metastasis from malignant melanoma. J Surg Case Rep. 2019;2:1-2. 
7. De Miguel M, Jimeno J, Vidal, Segura S, Lorente L. Tratamiento quirúrgico de rescate en paciente con melanoma metastásico con mutación BRAF V600E. Cir Esp 2018;96(10):656-66.

8. Reintgen DS, Thompson W, Garbutt J, Seigler HF. Radiologic, endoscopic, and surgical considerations of melanoma metastatic to the gastrointestinal tract. Surgery. 1984;9:635-9.

9. Silva S, Tenreiro N, Melo A, Lage J, Moreira H, Prospero F, et al. Metastatic melanoma: An unusual cause of gastrointestinal bleeding and intussusception-A-case report. Int J Surg Case Rep. 2018;53:144-6.
10. Zhou Ye, Zhe Yang, Shusen Zheng, Weilin Wang. Robot-assisted laparoscopic surgery for abdominal metastatic melanoma mimicking a gastrointestinal stromal tumor. Medicine (Baltimore). 2018; 97: e11207. Published online 2018 Jun 29. doi: 10.1097/ MD.0000000000011207. 